\title{
DR. EDUARDO BUSTOS-OBREGÓN (1937-2014)
}

Escribir del Dr. Eduardo Bustos-Obregón (1937-2014), es sin duda referirse en plenitud a la vida académica, la universidad y a la investigación científica. Se tituló de médico cirujano en la Universidad de Chile, y desde los inicios de su vida profesional mostró interés en la academia desde sus diversos ámbitos. Es así como realizó varias estadías de perfeccionamiento en el extranjero, como estudios de postgrado en la Universidad de Tulane (USA) y la Universidad McGill (Montreal, Canadá), mediante una beca de la Fundación Rockefeller, y en el Instituto de Anatomía de la Clínica Universitaria de Eppendorf (UKE, en Hamburgo, Alemania), gracias a una beca de la Fundación A. Von Humboldt.

Su vida a académica estuvo marcada por su gran y permanente interés en la formación de estudiantes de pre y postgrado, principalmente en las áreas de la biología celular relacionada con la reproducción, lo cual fue posible gracias a los numerosos proyectos de investigación otorgados por fundaciones nacionales e internacionales. Otro aspecto central y que se destaca en su quehacer académico fue el desarrollo y promoción de disciplinas como la Biología de la Reproducción, Gametología, Andrología y Toxicología desde diversos ángulos. Así, fue impulsor de programas académicos como el Magister en Biología de la Reproducción de la Facultad de Medicina de la Universidad de Chile y el Doctorado en Ciencias Morfológicas en la Universidad de La Frontera...entre muchos otros. Además, fue fundador de sociedades científicas como la Sociedad de Biología de la Reproducción y Desarrollo y Sociedad de Andrología y Gametología de Chile, de las cuales fue su presidente por varios años.

En este número especial de la revista International Journal of Medical and Surgical Sciences en homenaje al Dr. Eduardo BustosObregón, diversos colaboradores, discípulos y amigos, han querido contribuir exponiendo sus últimas investigaciones y/o realizando revisiones que abarcan diversos temas, todos ellos relacionados a la temática que desarrolló el Dr. Bustos-
Obregón durante su vida académica. Este número se desarrolla a través de tres ejes ó areas temáticas: la gónada masculina, el gameto y la fecundación y la patología del tracto reproductor masculino. En ellos se describen aspectos centrales de la fisiología testicular con especial énfasis en aspectos regulatorios y metabólicos de la espermatogénesis, la función espermática en su tránsito por el epidídimo y el tracto reproductor femenino, previo a la fecundación en mamíferos. También se destacan temas que abordan la acción de las hormonas en la capacidad reproductiva y los relacionados con la andrología. Finalmente, se incluyen aspectos de oncología en glándulas del sistema reproductivo y la participación de las células troncales tumorales en el desarrollo del cáncer de próstata. Estos trabajos representan a un importante número de miembros de la comunidad científica internacional y otros tantos de la comunidad nacional, vinculada a las universidades regionales, que siempre fueron de especial interés en el quehacer del Dr. Eduardo Bustos-Obregón.

Estas contribuciones están especialmente pensadas, seleccionadas y dirigidas a estudiantes de áreas asociadas a la reproducción masculina, quienes fueron siempre su especial dedicación.

Dra. Margarita Vega B. y Dr. Héctor R. Contreras Comité Académico del Magister en Ciencias Biológicas mención Biología de la Reproducción. Facultad de Medicina. Universidad de Chile.

Dra. Margarita Vega Blanco

Laboratorio de Endocrinología y Biología de la Reproducción. Hospital Clínico José Joaquín Aguirre (JJA). Universidad de Chile

Dr. Luis Valladares Boasi

Laboratorio de Hormonas y Receptores

Instituto de Nutrición y Tecnología de los Alimentos. Universidad de Chile

Dr. Héctor R. Contreras

Laboratorio de Andrología Celular y Molecular.

Facultad de Medicina. Universidad de Chile. 